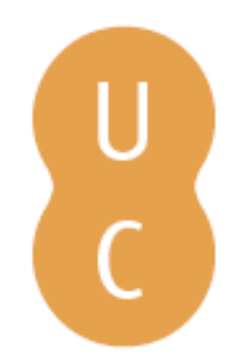

\title{
pompalina
}

\section{Las dimensiones filosófica y antropológica de la interpretación educativa}

$\begin{array}{ll}\text { Autor(es): } & \text { Peña Calvo, José Vicente; Rodríguez Neira, Teófilo; Gervilha Castillo, } \\ \text { Enrique; Pérez Alonso-Geta, Petra Ma. }\end{array}$

Publicado por: Imprensa da Universidade de Coimbra

URL

persistente: URI:http://hdl.handle.net/10316.2/38382

DOI: $\quad$ DOI:http://dx.doi.org/10.14195/978-989-26-0486-2_4

Accessed : $\quad$ 26-Apr-2023 14:13:19

A navegação consulta e descarregamento dos títulos inseridos nas Bibliotecas Digitais UC Digitalis, UC Pombalina e UC Impactum, pressupõem a aceitação plena e sem reservas dos Termos e Condições de Uso destas Bibliotecas Digitais, disponíveis em https://digitalis.uc.pt/pt-pt/termos.

Conforme exposto nos referidos Termos e Condições de Uso, o descarregamento de títulos de acesso restrito requer uma licença válida de autorização devendo o utilizador aceder ao(s) documento(s) a partir de um endereço de IP da instituição detentora da supramencionada licença.

Ao utilizador é apenas permitido o descarregamento para uso pessoal, pelo que o emprego do(s) título(s) descarregado(s) para outro fim, designadamente comercial, carece de autorização do respetivo autor ou editor da obra.

Na medida em que todas as obras da UC Digitalis se encontram protegidas pelo Código do Direito de Autor e Direitos Conexos e demais legislação aplicável, toda a cópia, parcial ou total, deste documento, nos casos em que é legalmente admitida, deverá conter ou fazer-se acompanhar por este aviso.

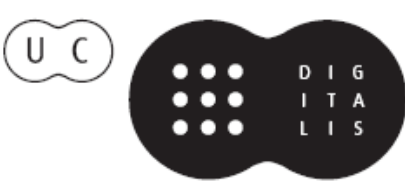


João Boavida

Ángel García del Dujo

Coordenação

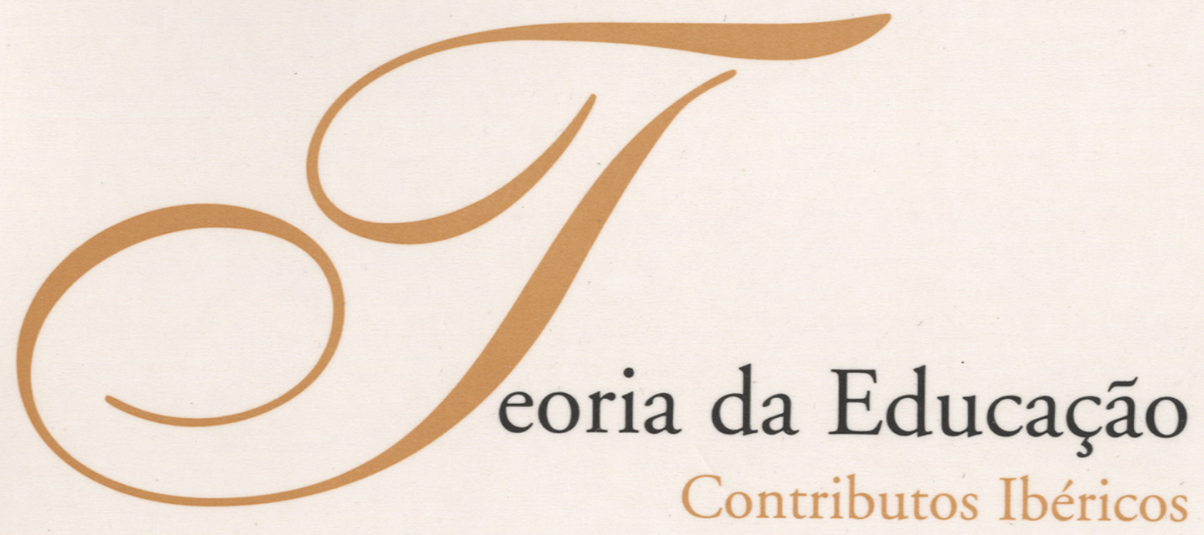


José Vicente Peña Calvo (coordinador)

Teófilo Rodríguez Neira

Universidad de Oviedo

Enrique Gervilha Castillo

Universidad de Granada

Petra Ma. Pérez Alonso-Geta

Universidad de Valencia

Las Dimensiones Fil o sófica y Antropológica de I a Inter pretación Educativa

El título general de estas pequeñas anotaciones necesita ser puntualmente aclarado, precisado. El término «dimensión» no quiere indicar que no sea posible organizar una serie de conocimientos sobre fenómenos determinados, fenómenos educativos, por ejemplo, sin ninguna referencia filosófica. De hecho, sobre la educación se han ido acumulando una serie amplia de conocimientos: conocimientos antropológicos, psicológicos, sociales, etc., que se presentan como resultado de estudios e investigaciones positivas. Miles de textos confirman estas afirmaciones. Lo que se pretende indicar, con la referencia que en estos apuntes se utiliza, es, únicamente, que los saberes acumulados sobre educación resultan incompletos sin una progresión filosófica en torno a ellos. Lo mismo se puede afirmar en relación con lo que representan las aportaciones antropológicas. Aunque, en este último caso, como indica la profesora Petra Ma. Pérez, la educación recibe un tratamiento que complementa el resto de lo que sobre la misma puede llegar a saberse. La Universidad de Oviedo, la de Granada y la de Valencia han dejado constancia en estas páginas, a través de los profesores que las 
enseñan, de la postura que defienden y del punto de vista desde el que imparten las disciplinas de Filosofía y Antropología de la Educación.

\section{Cambios y propuestas sobre Teoría de la Educación}

La Teoría de la Educación se ha ido construyendo en España como un campo de saber sometido a constantes revisiones y a orientaciones no siempre coincidentes. No se trata únicamente de que estemos en un mundo recorrido por revoluciones tecnológicas profundas y por cambios radicales de los «paradigmas» epistemológicos básicos. Sucede, más bien, que, al analizar los fenómenos educativos, encuanto fenómenos complejos, se los hace girar sobre elementos heterogéneos. Se puede comprobar, desde el punto de vista tradicional, que la educación ha disfrutado, durante siglos, de una fuerte dependencia religiosa, teológica, de una intensa influencia ideológica, de una incansable presión social y política, de una férrea influencia cultural y de una obligada filiación filosófica, 0 científica, o tecnológica, 0 axiológica, etc. Cada una de estas realidades ha presionado sobre las interpretaciones y sobre las prácticas educativas hasta límites insospechados. Y todavía continúan haciéndolo.

Se tiene la impresión de que no acaba de establecerse y concretarse un sistema categorial claro, bien definido, lo suficientemente amplio y flexible, que merezca la aprobación general y que pueda servir de punto de partida compartido y común. Quizá, semejante pretensión, dada la complejidad de lo que se está analizando y la presión histórica de los hechos a los que está sometido, sea imposible de alcanzar.

Por lo que respecta a las influencias filosóficas y a sus alternativas, la Teoría de la Educación se ha desarrollado, con deliberación y minuciosidad, como otro saber y otra forma de conocimiento distinto del exclusivamente filosófico. En más de una propuesta se puede constatar que la Teoría de la 
Educación se ha configurando como un saber contrapuesto al que puede corresponder a cualquier opción filosófica. El mismo nombre asignado y seleccionado implica una separación decidida y rotunda. En el volumen 16 de Teoría de la Educación. Revista Interuniversitária (2004), es decir, en el marco de la más estricta actualidad, los profesores Colom Cabellas y Rincón Verdera insistían en la necesaria independencia y autonomía de las cuestiones pedagógicas:

Creemos que una teoría que tenga en cuenta cuáles - y de qué tipo — son las realidades educativas más aproximará el saber pedagógico a la solución de los problemas educativos; en cambio, las teorías filosóficas de la educación — sobre todo las idealistas — al ontologizar la educación alejaron a la Pedagogía de su realidad inmediata. Creemos firmemente que la Pedagogía podría haberse desarrollado pareja a la Antropología, a la Psicología o a la Sociología, y, si su evolución no fue así, no se debió a su identidad epistemológica, sino a las influencias de corte espiritualista, que brevemente hemos querido reflejar al inicio de este trabajo. Más diremos, sin la influencia de los espiritualismos de Badén, hoy no sería necesaria la Teoría de la Educación, pues la Pedagogía podría haberse desarrollado paralelamente a las otras ciencias sociales (Colom y Rincón Verdera, 2004, 41).

La posición está claramente delimitada y ha sido rigurosamente aplicada por los teóricos de la educación. Existe un marco y una orientación decidida en la que participan, pese a la diversidad de los desarrollos llevados a cabo, bastantes pedagogos de diferentes nacionalidades. Los profesores españoles que han dedicado estudios a estas cuestiones, como puede comprobarse por los nombres y las aportaciones que están presentes en este libro (sin necesidad de recurrir a la abundante bibliografía que han ido generando), son numerosos y, ya que están presentes en estas páginas, no es necesaria su repetición, sin acudir a otras disculpas y justificaciones. Una mera presentación de los diferentes puntos de vista nos llevaría a la redacción de tantas páginas como las que aquí pueden aparecer.

La pugna y el trabajo sobre estas cuestiones no han cesado. En parte, debido a las dificultades internas y externas del problema. Debido, también, a los múltiples empeños encaminados a reducir unilateralmente las cuestiones. 
De hecho, frente a todos los esfuerzos orientados a la configuración de una Teoría de la Educación consistente en el conocimiento de los fenómenos educativos, de las regularidades que les corresponden y de los criterios prácticos con los que deben promoverse e implantarse, no cesan de aparecer interpretaciones y soluciones asentadas en supuestos diferentes, en criterios ideológicos o en otras posiciones semejantes. Al mismo tiempo, como se puede constatar fácilmente, las distintas filosofías emergentes, desde el estructuralismo al deconstruccionismo, desde el idealismo al existencialismo y al narrativismo, desde el furor analítico al axiologicismo, etc., no cesan de intervenir en los temas educativos e interpretarlos desde sus respetivas posiciones. Esta proliferación infatigable enturbia permanentemente los problemas.

2. La interpretación filosófica de la educación y su complementariedad gnoseológica en relación con el resto de saberes sobre los hechos educativos

Aun admitiendo y dando por válida la configuración de una Teoría de la Educación de corte cientifista, respetando todos los estudios producidos en esta dirección, es necesario reconocer dos cuestiones fundamentales:

a) Todo sistema filosófico, cerrado 0 abierto, directa 0 indirectamente, termina siempre en una interpretación de la educación. Esto ha sido así, está siendo de esta manera y continuará realizándose de semejante modo, independientemente de las voluntades concretas y de las críticas que sus soluciones puedan desencadenar, b) Los fenómenos educativos, lo mismo que todos los campos establecidos y delimitados de fenómenos físicos, políticos, sociales, artísticos, etc, son susceptibles de ser tratados, analizados y estudiados desde un punto de vista filosófico. Existen filosofías regionales: filosofía del arte, filosofía de las acciones políticas, filosofía de las prácticas tecnológicas, filosofía del lenguaje, etc., que han adquirido gran predicamento 
e influencia. Nadie puede negar la existencia de una filosofía de la educación real y fructífera a no ser que se empeñe en negar una realidad histórica y una disciplina pedagógica profesionalizada y vigente.

No es necesario detenerse en una fundamentación pormenorizada de estos dos puntos. Con relación al primero, pese a reconocer el exceso en el que incurre, se puede recordar la famosa afirmación de Dewey: «Incluso podría definirse a la filosofía como la teoría general de la educación». El hecho es que todos los sistemas filosóficos, de una forma explícita 0 implícita, como decíamos, terminan en interpretaciones de la educación y de su significado, interpretaciones que han alimentado teorías de la educación y aplicaciones de la misma. Desde Platón a Foucault, desde Aristóteles a Derrida, desde Nietzsche a Wittgenstein, desde Arendt a Schlick, todos han abordado e inspirado concepciones y orientaciones de la educación que complementan cuantos estudios se hagan o puedan hacerse sobre los fenómenos educativos. Incluso el propósito de desmarcar la Teoría de la Educación de los supuestos filosóficos en los que con frecuencia se ha apoyado puede ser comprendido y analizado desde planteamientos epistemológicos no exentos de inspiración filosófica.

La explicación de la inevitable participación de los sistemas filosóficos en cuestiones educativas y en una concepción del ordenamiento en el que se lleva a cabo su desarrollo, entre otros aspectos y razones, se encuentra en el hecho de que cualquier doctrina filosófica general termina, como afirmara Kant, en una concepción del hombre. Y, según se ha repetido incesantemente, todo tipo de educación entraña una cierta comprensión y visión de la que es el ser humano y de lo que debe llegar a ser.

El segundo aspecto merece también alguna presentación, aunque sea resumida y simplificada. Es innegable que los fenómenos educativos son susceptibles de ser filosóficamente estudiados. Entre otras razones, porque así se está haciendo. Passmore, Ryle, Woods, Barrow, Hamlym, Hirst, Kingsley Price, Langford, Peters, Scheffler, Warnock, por citar sólo un puñado de nombres conocidos, prescindiendo deliberadamente de un amplio número de pensadores internacionales y de los profesores españoles que se dedican 
a impartir Filosofía de la Educación como disciplina, profesores que Gil Colomer logró agrupar durante años y que han dejado contribuciones valiosas, todos ellos confirman esta realidad. Las implicaciones han sido largamente debatidas. No obstante, como sucede con los conocimientos que el hombre ha ido acumulando sobre el mundo, sobre la sociedad, sobre la vida y sobre sí mismo, todos estos saberes remiten siempre a un conjunto de categorías universales y últimas desde las que sea posible conquistar una visión y una síntesis fundamental de los mismos. Esta tarea de corte filosófico ofrece un complemento imprescindible para entender lo que realmente significan y son los datos que allí se analicen y descifren. Esta afirmación es válida para todos los ámbitos de conocimiento y para las teorías que en torno a esos ámbitos se formulen. De un modo más imperioso se puede mantener una postura semejante para todos los conocimientos que sobre las prácticas educativas lleguen a formularse. Una teoría de la educación, en cuanto conocimiento de los hechos educativos, está incompleta sin una filosofía de la educación.

Sucede, no obstante, que al hablar de la filosofía como un saber que se consigue después de haberse desarrollado los conocimientos concretos, no se puede entender al modo de una filosofía ya dada e incontrovertible. La filosofía, como el conjunto de los saberes humanos, se desenvuelve, cambia, se modifica y adquiere formulaciones diversas. El progreso y el desarrollo consisten, precisamente, en la capacidad de los humanos para reformular sus propias concepciones del mundo y de la vida.

\section{Filosofías de la educación}

A lo largo de la historia, como es natural, se han ido formulando diversas concepciones filosóficas, no sólo sistemas distintos y enfrentados entre sí, sino filosofías distintas sobre un mismo conjunto de hechos. Estas filosofías 
se han clasificado en atención a los sistemas de los que surgen o de aquellos a los que recurren para su establecimiento y acreditación. Pero también se pueden agrupar en función de los elementos que recogen del sistema al que se refieren y desde los que intentan definir los procesos generales dentro los cuales se producen aquellos elementos. Cabe señalar, desde este último punto de vista, las siguientes corrientes y alternativas:

a) Existe un conjunto de propuestas filosóficas encaminadas a defender y promover el papel que deben representar los sujetos de la enseñanza y de la educación, los aprendices, en el sentido más amplio del término. En toda práctica educativa hay individuos concretos que son «educados» a través de actividades e influencias de todo tipo. Pues bien, abundan las corrientes filosóficas que convierten las características, atribuidas a esos sujetos, en factores reguladores de la totalidad del proceso educativo. Estas corrientes han recorrido un trayecto largo y de gran influencia. En esta dirección se encuentra Rousseau, por ejemplo, con todo un ejército de seguidores. A esta orientación pertenecen todos los autores de la Pedagogía Institucional... Incluso Rogers, con su apología de las experiencias vivenciales y sus terapias psicológicas, con esa grandilocuencia que lo condujo a una visión negativa de la enseñanza y a un radicalismo sorprendente y excesivo, se mantiene en esta línea.

b) En lado opuesto, enfrentadas a las concepciones anteriores, dando pábulo a una gran variedad de interpretaciones educativas, aparecen todas las filosofías que tienden a exaltar la dimensión social de la educación y la enseñanza. Desde todas las corrientes marxistas al holismo propugnado por Durkheim y a cuantos autores, pasados y presentes, insisten en el carácter esencial de las relaciones sociales, se ha ido formulando una verdadera multitud de alternativas. Recuérdese, como simple ejemplo, el hechizo que durante algún tiempo ejercieron autores como Berstein 0 Niklas Luhmann.

c) Durante los últimos años se han sucedido no pocas concepciones encaminadas a propagar y extender prácticas educativas fundadas en 
planteamientos axiológicos, en criterios de valor. Un mundo de filosofías, cada una de ellas tirando hacia una interpretación de los valores de distinto corte pragmático, se ha ido proponiendo sin tregua. Viejos y nuevos sistemas concurren en este ámbito. Algunas de las corrientes han adquirido una gran resonancia. En esta línea habría que situar muchas de las alternativas ofrecidas por algunos representantes de la Investigación-Acción en sus diferentes formulaciones. El camino está abierto y todos los estudiosos de estas cuestiones conocen la multitud de variantes que se han ido proponiendo y se continúan formulando.

d) Otra serie de alternativas filosóficas se ha ido promoviendo desde el papel que se atribuye a los agentes directos e indirectos de la educación. Las sucesivas interpretaciones del poder político, de los sistemas productivos, de las culturas, de los profesionales de la enseñanza y de sus roles, han llenado cientos de páginas y han generado multitud de interpretaciones y de referencias prácticas. Al mismo tiempo, a medida que los medios (el medio es el mensaje) han ido invadiendo el mundo contemporáneo, se han sucedido las corrientes encaminadas a su estudio y a su exégesis.

e) Por otro lado, la dimensión psicológica de la enseñanza se ha convertido en una fuente inagotable de teorías y de filosofías de la educación. El conductismo, el constructivismo, el cognitivismo, etc., han contribuido a reformar escuelas y programas, han dado lugar a interpretaciones generales y a debates intelectuales que terminaron afectando a todo el sistema educativo. En la medida en que se convirtieron en explicaciones globales, se fueron transformando en auténticas filosofías de la educación, más allá del modelo psicológico del que brotaron y en el que se fundamentaron, etc.

Después de este pequeño recorrido, de esta somera presentación de diferentes concepciones, se puede tener la impresión de que se han agotado todas las posibilidades de llevar a cabo nuevos análisis. No obstante, ha sido factible incorporar aspectos todavía no explícitos en las anteriores líneas de desarrollo. En concreto, si pensamos que toda educación es, realmente, un proceso, una secuencia de actos con los que se pretende conseguir unos 
resultados determinados, entonces, lo que mejor puede contribuir a justificar la educación es una rigurosa formulación de los fines a los que se aspira. No se olvide que, como se ha dicho, da característica esencial de la inteligencia humana es la invención y la promulgación de fines». Toda la vida humana está dirigida, gobernada y organizada en función de los fines que somos capaces de proponernos. Y la educación, en cuanto proceso en el que la vida misma se pone en juego, es un ejercicio que sólo podrá entenderse desde los fines que se quieren alcanzar. Los fines abren un campo fértil desde el que se puede reconstruir toda la historia de la educación, crean un orden apropiado para promover los desarrollos más eficaces.

No es necesario formular un finalismo ontológico que podría desvirtuar todo el análisis general. La tesis de estudio que se ha abierto en alguna universidad, como se ha escrito y defendido en Congresos y Seminarios, es muy sencilla:

La educación pertenece al conjunto de acciones que los individuos de una sociedad ejercen unos sobre otros, o sobre sí mismos, para lograr una vida más perfecta y plena. Los fines, en este sentido, son un requisito imprescindible y esencial de las acciones, tanto para su determinabilidad como para su comprensión. Es decir, las acciones pierden su carácter de humanas cuando dejan de estar sometidas a propósitos y a metas intencional, deliberada y racionalmente propuestas. Y, al mismo tiempo, se nos escapa su intelección y conocimiento cuando prescindimos de los fines bajo cuya dirección han sido declaradas y propuestas (Rodríguez Neira, 1999a, 134).

La tesis, pese a dejarla en un simple enunciado sin ningún desarrollo, se defiende frente a todas las alternativas que, bajo el signo de la modernidad 0 del progreso, se han caracterizado por un antifinalismo declarado y expreso. Los fines y su formulación han sido considerados como restos de un dogmatismo anticuado y fuera del tiempo, como contrarios a los criterios más actuales de la emancipación. Los fines, desde esta perspectiva, fueron sustituidos por funciones. La educación, en este sentido, debería establecerse para cumplir unas funciones concretas y específicas. Recuperar una concepción de los fines ha significado aproximarnos a esa dimensión de las acciones sin la cual las acciones mismas pierden la categoría de humanas. 
Toda la educación, las etapas, los niveles, los programas, la organización escolar, ha sido estudiada desde este planteamiento filosófico y desde esta peculiar representación.

El significado de la Antropología y una reafirmación de lo que representa la Filosofía de la Educación están presentes en estas páginas a través de las aportaciones enviadas por los representantes de la Universidad de Granada y de Valencia. Sus textos sirven de testimonio general y de verificación de la orientación que allí dan a estas materias.

\section{Filosofía y filosofías de la educación: otras aclaraciones y precisiones}

El vocablo filosofía se encuentra cargado de múltiples sentidos 0 rostros $y$, en consecuencia, es adecuado su uso lo mismo en singular que en plural, pues todas las filosofías, siendo plurales, coinciden en ser filosofía. En referencia a la educación, el plural «filosofías» quizás exprese mejor la multiplicidad de modelos o paradigmas inherentes al saber filosófico y, en consecuencia, a la Filosofía de la Educación.

Ningún filósofo, ni escuela 0 corriente de pensamiento se ha atrevido a presentar su concepción del mundo y de la vida como única y verdadera, así como ningún modelo educativo ha logrado imponerse sobre los demás como el mejor. Cuando en este ámbito se habla de la verdad o del valor siempre son referidos a personas o a colectivos. La historia es fiel reflejo de esta pluralidad. Si ha existido algún predominio paradigmático, ha sido siempre temporal y circunstancial. Hay múltiples filosofías de la educación, como hay muchas filosofías de la vida:

No hay una filosofía de la educación, sino múltiples y, además, en insoslayable mudanza todas ellas. Si de unidad puede hablarse en tales menesteres, se referirá siempre a los prolegómenos o condiciones a toda posible filosofía de la educación; por lo demás, fuera de tal ocupación casi tautológica, nos situamos en el reino de la diversidad manifiesta, en el reino de la 'doxa' (Fullat, 1992, 11). 
Ello justifica la diversidad de enfoques, así como la multiplicidad de funciones que, por su naturaleza, ha de darse y desempeñar, antes como ahora, la Filosofía de la Educación, en cuanto ámbito de reflexión filosófica propia de este saber, tanto desde el punto de vista histórico como metodológico. Siendo su finalidad fundamental el conocimiento filosófico de la educación, el modo de hacer realidad tal finalidad en las distintas universidades, tanto en su contenido docente como investigador, es altamente plural.

\section{Perfiles actuales de desarrollo}

La pluralidad indicada es manifiesta desde el inicio de la Filosofía de la Educación en España. Las publicaciones de los especialistas de esta materia, sobre todo a partir del año 1988, así como la temática de los cinco congresos internacionales, son prueba de ello. Buen número de universidades españolas, sin que tal materia sea troncal, han optado por la Filosofía de la Educación como materia de estudio curricular y tema de investigación.

En la Universidad de Granada la Teoría de la Educación coexiste con la Filosofía de la Educación, dos dimensiones o direcciones de la educación: una filosófica y otra más tecnológica. En consecuencia, pues, la docencia e investigación gira en torno a dos asignaturas denominadas Filosofía de la educación, de carácter obligatorio, y Teoría de la educación, troncal, en los estudios de Pedagogía. El grupo de profesores que se ocupan de tales materias forman parte del Grupo de Investigación denominado «Valores Emergentes y Educación Social»(HUM-580), reconocido y subvencionado por la Junta de Andalucía desde el año 1995.

Nuestra aportación, filosófica y científica, se centra fundamentalmente en el campo de la axiología educativa. En el ámbito más filosófico, nos hemos ocupado de ciertos temas alusivos al profesorado, alumnos, textos 
legislativos, etc. Y en el campo científico, la elaboración de tests (sobre la persona, cuerpo, sexualidad, ancianos, etc.) y su aplicación a colectivos diversos nos ha deparado una fuente de información rica y amplia, algunos de cuyos conocimientos ya se han dado a conocer y otros se encuentran en vías de publicación.

Las tesis doctorales sobre temas más filosóficos son significativas al respecto. Algunas de estas investigaciones han sido realizadas fuera del territorio español, Argentina, Perú y México, y cuyos títulos son expresión ilustrativa de su contenido:

a) Unas se han ocupado de los valores de profesores y alumnos en los países indicados: «Los valores del profesorado en formación y su incidencia educativa», «El mundo actual. Valores e implicaciones educativas de los alumnos al finalizar la escolaridad obligatoria en Mar de la Plata (Argentina)», «El valor sexual en la educación integral de la persona. Un análisis estimativo en la Facultad de Ciencias de la Educación de Granada», «Crisis de valores e implicaciones educativas en el proceso de socialización de los jóvenes en la Universidad Privada de Tacna (Perú)».

b) Otras han versado sobre valores específicos como la paz y la tolerancia: «El concepto de educación en Krishnamurti y sus implicación en la educación para la paz», «La tolerancia como valor: clarificación conceptual e implicaciones educativas».

c) Otras han investigado los valores, expresos e implícitos, en textos legislativos, idearios, programas y métodos: «Los valores de la LOGSE y sus repercusiones educativas», «Los valores en los métodos de alfabetización de adultos en España», «Modelo educativo según los valores de los idearios granadinos», «Formación Cívica y Ética: valores e implicaciones educativas (México)».

d) Otros trabajos, finalmente, han estudiado las instituciones y el modelo de educación integral: «El Seminario de Maestros del Ave María. Un compromiso por la renovación social y educativa», «Análisis de un modelo de educación integral». 
El desarrollo de la actividad investigadora de este grupo ha dado lugar a un conjunto amplio de publicaciones: libros, artículos, actas de congresos nacionales e internacionales, etc. en cuya base reside la relación valores-educación y cuya extensa relación puede consultarse en www.ugr.es/ vees/.

\section{Implicaciones e influencias en la Teoría de la Educación}

Acorde con lo indicado, la aportación de la Universidad de Granada a la Teoría de la Educación está en relación al concepto mismo de teoría: su contenido, función y relación con las Ciencias de la Educación.

Actualmente, entre los especialistas, existe cada vez un mayor consenso en sostener que la Teoría de la Educación ha de ser una teoría tecnológica susceptible de aplicar a cualquier tipo de paradigma educativo, por lo que lo tecnológico es la forma de entender cualquier teoría o paradigma educativo (Colom y Núñez, 2001, 37-38). Se trata de saber hacer, de conocer para mejorar la práctica educativa, por lo que la esencia de la Teoría de la Educación está en su pragmatismo. En consecuencia, pues, cuando el conocimiento teórico no posea aplicabilidad directa o inmediata a situaciones educativas, nunca se integrará como conocimiento propio de la Teoría de la Educación (Ibidem, 60).

Desde esta concepción, ¿qué aporta la Filosofía de la Educación a la mejora directa de la práctica educativa? La Filosofía de la Educación, en cuanto filosofía práctica, ¿qué aplicabilidad otorga a la educación? ¿Es la Filosofía de la Educación un saber alejado de la vida? ¿Puede la Teoría de la Educación, para llevar a buen término su tarea, prescindir de la Filosofía de la Educación? Las palabras de Baltasar Gracián, en El Criticón, son ilustrativas de la función de la filosofía, y más aún de la Filosofía de la Educación: «el saber de las cosas y no obrallas, eso no es ser filósofo, sino gramático». 
Sin Filosofía de la Educación quedan sin respuesta, al menos de modo explícito, interrogantes tales como ¿qué elegir?, ¿es posible educar sin modelo último de referencia?, ¿por qué un modelo y no otro?, ¿quién decide y quién debe decidir?, ¿se debe imponer al educando una opción contra su voluntad?, ¿deben los políticos imponer su modelo ideológico porque gozan de mayoría?, ¿qué dimensiones humanas hemos de educar?, ¿quienes han de educar?, ¿es posible justificar cuál es la mejor educación?, jes posible la neutralidad?, ¿son indiferentes los medios?... Silencios que, quizás, interesen a ciertos políticos, manipuladores, adoctrinadores, 0 a quienes quieren reducir el ser humano a ciencia y tecnología, pero no a los formadores 0 educadores de la integridad de la persona.

Una Teoría de la Educación, por ser de la educación y no otra teoría - y, por tanto, de lo humano y sobre lo humano — jamás, pensamos, podrá prescindir de la Filosofía, al menos de ciertos temas, propiamente filosóficos, como 1) axiología y teleología: se podrá discutir qué valores, pero no el fundamento axiológico de la educación; pueden darse divergencias en torno a cómo entender los fines, pero no en que toda acción educativa se orienta a un fin, aunque tal fin sea la carencia de fin preestablecido. Quien se interroga por la Filosofía de la Educación está preguntándose acerca de qué dirección 0 sentido darle a la labor que realiza, pues la educación es un fenómeno demasiado complejo como para reducirlo a la racionalidad instrumental. 2) Ética, por cuanto las cuestiones morales son inseparables de la persona y, por lo mismo, de la educación. Debate, siempre abierto, dada la pluralidad de concepciones antropológico-morales, pero imprescindible. 3) Clarificación del lenguaje, dado el actual poder de la palabra, el mayor don y también el mayor riesgo. Palabras como progreso, libertad, humanización, autonomía, etc., esenciales a la educación, necesitan precisar su sentido para no incurrir en la ambigüedad, dificultando la comunicación y posibilitando la manipulación.

La Teoría de la Educación, pues, por ser educación, necesita de la Filosofía de la Educación, por cuanto la ciencia no agota la realidad humana. 
Ciencia y tecnología, siendo necesarias, son insuficientes para educar, pues la complejidad de la formación humana es irreductible a la ciencia, el ser humano trasciende la ciencia, es más que ciencia.

Hoy, corren paralelos el prestigio del vocablo «teoría» y el desprestigio de «filosofía». El valor de la filosofía de siglos atrás quedó en entredicho tras la ruptura renacentista (Scienza Nuova) y el divorcio positivista. A quienes hoy nos dedicamos a la Filosofía de la Educación nos resulta difícil persuadir a nuestros alumnos —ya veces también a algunos miembros de la comunidad científica —-de algo que antes era innecesario: el valor de la filosofía. Ésta frecuentemente es cuestionada, rodeada de dudas y, a veces, hasta de desprecio. Entre las múltiples causas que inciden en esta situación de «enfermedad» y debilidad de la misma, pueden destacarse las siguientes:

a) La identificación de la racionalidad en general con la racionalidad instrumental o tecnológica. La gravedad de esta racionalidad reside en haber devorado otras formas de racionalidad, reduciendo todo intento de racionalidad a la racionalidad instrumental. De este modo, se niega la racionalidad moral, estética, mística 0 religiosa... de «lógicas» distintas y axiología antagónica. El desarrollo humano, conducido por la racionalidad tecnológica 0 instrumental, deja sin respuesta cuestiones esenciales de la educación, cuyo fundamento fue y sigue siendo la pregunta, tan antigua como nueva, iqué es ser persona?, ¿quién es el ser humano?

b) La importancia de lo útil, lo práctico e inmediato, es tal que, para no pocos, queda sin valor cuanto no cae bajo su influencia. El problema no es nuevo. Ya Bertrand Russell alertaba del peligro del «hombre práctico» que sólo reconoce necesidades materiales, olvidando las necesidades del espíritu:

Si todos los hombres vivieran bien, si la pobreza y la enfermedad hubieran sido reducidas al mínimo posible, quedaría todavía mucho por hacer para producir una sociedad estimable; pues, en el mundo actual, los bienes del espíritu son por 10 menos tan importantes como los del cuerpo. El valor de la filosofía debe hallarse exclusivamente entre los bienes del espíritu, y sólo los que no son indiferentes a estos bienes pueden llegar a la persuasión de que estudiar filosofía no es perder el tiempo (Russel, 1972, 30). 
c) A esta escasez de los bienes del espíritu se suma la falta de apoyo político. Filosofía y política han sido, tradicionalmente, un matrimonio siempre en conflicto entre la razón filosófica y la sin-razón política. Desde que Sócrates fue condenado a beber la cicuta hasta la actualidad, la enemiga de la filosofía, más aún que la ciencia o la técnica, ha sido la polííca, ya que cuando los políticos no pueden controlar el discurso filosófico terminan por suprimirlo, bien a base de censura 0 por medio de leyes y decretos. De este modo, alejan la política de la enseñanza 0 bien la «domestican», para que los adolescentes no aprendan a pensar por sí mismos críticamente en contra de los poderes constituidos (Gervilla, 1990).

Es significativo al respecto el interés de un buen sector del profesorado por reivindicar la importancia de las «humanidades» tras su gradual pérdida en las últimas reformas del sistema educativo. Y ello parece aún más antagónico cuando, de modo simultáneo, se proclama que es preferible la formación a la instrucción, que los alumnos, en esta etapa educativa, más que aprender contenidos, han de aprender a aprender, a pensar, a criticar...

La filosofía y, en nuestro caso, la Filosofía de la Educación, ¿carece de sentido en esta sociedad tecnificada 0 es más necesaria que nunca? ¿Ha muerto, tras largos siglos de existencia, por carecer hoy de valor, o la han asesinado por no interesar a quienes ostentan el poder político 0 académico? ¿Interesa hoy, de verdad y no de palabra, al sistema educativo la formación de personas reflexivas y críticas, 0 es más conveniente el silencio y la pasividad de los ciudadanos?

\section{El papel de la Antropología en la Teoría de la Educación}

El papel de la antropología cultural en la Teoría de la Educación se resuelve en el desarrollo de un conocimiento que permite obtener «datos 
relevantes» sobre los cuales apoyar la toma de decisiones educativas. No en vano, en la ciencia actual, la aportación teórica a la «toma de decisiones» es un criterio epistemológico básico de justificación de 10 científico. En este sentido, entendemos que cualquier reflexión teórica ha de partir de datos, como también, siguiendo a Geertz $(1996,130)$, que es necesario trabajar sobre datos reales, de individuos reales, en culturas reales. Geertz es un antropólogo empirista y resuelve su propuesta en clave de ciencia empírica. Nuestro trabajo antropológico es también educativo y, en educación, en ocasiones, hay que tomar decisiones en base a datos reales y, en algunos casos, de explicaciones probabilísimas contrastadas. Desde esta posición, que no pretende ser la única opción posible, debe entenderse, nuestra aportación a la teoría desde la Antropología de la Educación.

La Antropología de la Educación se ocupa, desde un enfoque antropológico, de los procesos y problemas educativos. Desde los años sesenta vive una época floreciente; sin embargo, en una revisión de la investigación al uso, se observa que todavía no constituye un área bien definida. En palabras de Goetz y Le Compte:

En esencia, constituye una síntesis interdisciplinar emergente, al practicarla investigadores de distintas tradiciones. Sin embargo, estos no han llegado a un consenso respecto a cuál debe ser su alcance y su método (1988, 42-43).

De hecho, dentro del campo de la Antropología de la Educación, aún sin explicitar, se han utilizado tradicionalmente marcos teóricos del ámbito de las ciencias sociales — sociología y psicología — fundamentalmente. Los etnógrafos no comparten una única definición de cultura y en sus trabajos han sido influenciados por otras disciplinas (Psicología Educativa, Medicina, Sociología de la Educación...) y por orientaciones de investigación de corte positivista. Por eso, sus aproximaciones metodológicas específicas varían considerablemente (Jacob, 1987). Además, en el campo de la educación, especialmente a partir de la década de los ochenta, se han desarrollado estudios etnográficos que son virtualmente idénticos a los que llevan a cabo los antropólogos (Goetz y Le Compte, 1988), alentados por una práctica que 
conecta con toda una filosofía de investigación educativa —en la década de los ochenta — dominante: la idea de «profesor investigador» que debe adoptar una postura de indagación sobre su práctica.

Por otra parte, no hay que olvidar que, aun contando con la influencia que el marco teórico tiene en la selección de estrategias para la recogida de datos, se observa en la investigación social una tendencia ascendente a la utilización ecléctica de metodologías. Por ejemplo, en el paradigma de la definición social (teoría de la acción, interaccionismo simbólico, etnometodología, etc...) el método de la observación participante y no participante es definidor de los partidarios de este paradigma, muchos de los cuales utilizan también el método del cuestionario-entrevista (Ritzer, 1993, 603). Siendo, en el campo de la antropología, la técnica etnográfica la metodología básica.

La Antropología de la Educación, desde nuestra perspectiva, debe centrarse en el estudio de los procesos de transmisión-adquisición cultural, pero, además, ha de descender a las diversas «clases» y grupos de individuos, descender a lo particular, a lo circunstanciado y concreto: a conocer y comparar cómo son los valores, motivaciones y pautas de los que aprenden y se educan, a conocer los contextos en los que se lleva a cabo la enculturación (familia, escuela, etc.) y al modo en que todo ello se desarrolla y cambia en el tiempo o de un lugar a otro. Con atención muy especial a la interacción existente entre cultura, educación e individuo como un todo.

Esta forma de entender la Antropología de la Educación hunde sus raíces en la década de los 30, cuando se consolida la corriente Cultura y Personalidad, corriente clave que orienta el nacimiento de la Antropología de la Educación. En esta época, junto a los estudios de la adolescencia, interesan especialmente a los antropólogos, F. Boas y sus discípulos (la misma M. Mead y R. Benedict), los primeros años de vida del niño, ya que para ellos, a través de los procesos educativos que configuran la crianza en la primera infancia, se evidencian ciertos determinantes en la conformación de la personalidad del individuo. Con estos estudios, la educación pasa a 
ser el eje fundamental de sus intereses y objetivos, por el papel que juega en la confluencia de cultura y personalidad (Colom y Janer, 1995; Colom, 2005).

Atendiendo a los anteriores supuestos, la investigación en Antropología de la Educación debe comenzar con el planteamiento de un problema 0 grupo de problemas que hay que estudiar. A veces el punto de partida es una teoría educativa bien fundamentada, de donde se extraen una serie de hipótesis que hay que verificar. Otras veces, se trata de generar hipótesis acerca de una situación, las cuales pueden permitir avances considerables en la clasificación de los problemas y el desarrollo educativo de investigaciones futuras.

Desde la perspectiva que asumimos, si bien la etnografía es la técnica básica que nos permite observar, escuchar, comparar y describir, no entendemos la misma como un paradigma alternativo a la investigación cuantitativa, sino más bien como un método, con unas determinadas ventajas en relación a la investigación educativa en antropología y en ningún modo antagónico, sino muchas veces complementario, de la investigación cuantitativa, pudiendo proporcionar, en este sentido, evidencias sobre la plausibilidad de diferentes líneas de análisis que pueden llevarse a cabo posteriormente en la investigación 0 si las regularidades observadas etnográficamente se confirman en toda la población. De igual forma que puede contribuir a la formulación de hipótesis a contrastar en diseños de investigación controlada cuantitativa.

De acuerdo con lo expuesto anteriormente, nuestra aportación desde la Antropología de la Educación tiene como eje central dos líneas fundamentales de investigación, básica y aplicada. Corresponden a distintos proyectos que se inscriben en las mismas y que nos han permitido conocer la realidad educativa para, sobre esta base, implementar acciones de mejora; 0 , en su caso, contrastar las bases teóricas de algunos supuestos. En definitiva, el análisis y estudio de los entornos y procesos de enculturación (pautas de crianza, educación y socialización) se ha llevado a cabo utilizando la 
metodología cualitativa, etnográfica, y a veces también la cuantitativa, para acercarnos al conocimiento de la infancia y adolescencia y los entornos donde se socializa y educa.

Dentro de la investigación básica cabría citar las realizadas dentro del contexto familiar en los estudios de Pérez Alonso-Geta y Cánovas Leonhardt, «Valores y Pautas de Crianza Familiar (0-6 años)» y «Valores y Pautas de Interacción Familiar en la Adolescencia (13-18 años)», que, junto a los datos aportados por la investigación del seguimiento del entorno familiar (7-13 años) actualmente en curso, nos está permitiendo elaborar un modelo teórico de «estilos educativos familiares» (Baumrind 1996; MacCaby-Martín, 1983) adaptado a nuestro entorno social.

Los datos cualitativos (etnográficos) y cuantitativos de estas investigaciones han permitido definir una serie de tendencias 0 estilos educativos familiares en España, dentro de los cuales es posible ubicar la acción educativa de los padres y madres con sus hijos (permisivos, comprometidos en el logro, desorientados...), así como el análisis comparado con los entornos exclusivamente rurales (Valle del Pas) nos permitió constatar la diferencia en valores, refuerzos y pautas de acción en la educación familiar en función del carácter más o menos comunitarista de los contextos familiares.

En el ámbito escolar, hemos llevado a cabo una investigación sobre «la cultura de la norma en los escolares españoles»; en ella pudimos conocer la motivación de los escolares para el seguimiento y la transgresión de las normas (coger algo que no es suyo, mentir...) y los sentimientos que en ellos genera el seguimiento y la transgresión. En concreto, pudimos comprobar que son los sentimientos de malestar que anticipan ante el conocimiento por los demás de su transgresión lo que les motiva, en mayor medida, a evitar la transgresión.

Dentro también de esta línea de investigación básica, hemos realizado, subvencionados por diferentes empresas, estudios como «el papel socializador del juego y el juguete», «los hábitos lectores en la literatura infantil» 0 «el efecto socializador de la TV en niños y adolescentes de la Comunidad Valenciana». 
Este último estudio ha puesto de manifiesto datos relevantes acerca del papel que la TV desempeña (ocio, aprendizaje ...) en la infancia y adolescencia y sus efectos en la percepción y construcción de la realidad social. Nos ha permitido conocer los efectos cognitivos, afectivos y comportamentales de la TV en los más jóvenes, a través de los procesos de identificación que desarrollan, ya que son capaces de enlazar la situación anímica de los modelos con su realidad, llevando a cabo de hecho una socialización anticipatoria que en gran medida les hace quemar etapas y reducir su infancia.

Por su parte, «Los valores de los niños españoles» (1992) nos permitió conocer la realidad infantil y sus contextos de socialización (familia, escuela, juego, tiempo libre...). Este trabajo se replicó en Andorra en el estudio que realizamos (1996) sobre «Valores y estilos de vida de la infancia en Andorra». En él, además de estudiar los valores y estilos de vida de los niños que viven en Andorra, se pusieron de relieve las discontinuidades que sufre la escolarización de los escolares con el paso del primer al segundo ciclo de Primaria.

El análisis comparado con los niños escolarizados en la misma edad en España (Pérez Alonso-Geta y otros, 1992) reveló que la transición en la escolarización de primaria ( $\mathrm{I}^{\circ}$ a $2^{\circ}$ ciclo) era mucho más traumática en el Principado de Andorra que en España. A partir de estos datos se puso en marcha una investigación, dentro de la línea de investigación aplicada, para una determinada institución educativa de Andorra, que ha preferido permanecer en el anonimato.

Esta práctica antropológica de aplicación, que nosotras consideramos fundamental en Antropología de la Educación, exige que las proposiciones teóricas de las que se parte estén muy delimitadas y sean inequívocas en sus planteamientos. Sólo así es posible la corroboración de las hipótesis y, en su caso, la apertura a nuevas ideas y propuestas que permiten analizar las consecuencias no previstas, más si cabe en el análisis de un tema tan complejo como las discontinuidades en la transición de un ciclo a otro de educación primaria, ya que son muchas y de muy diversa índole las variables 
que intervienen. En el Principado de Andorra vienen a ser como «cambios» culturales de salida de una cultura y entrada en otra nueva, con episodios que incluso podríamos calificar de «aculturación», ya que el cambio supone a veces la inmersión en un tipo de escolarización diferente (andorrana, francesa, española...) de la de partida.

En la transición al segundo ciclo de primaria, en Andorra, pudimos constatar alteraciones en las siguientes categorías: cambios de orientación cultural y linguística, cambio de centro, cambio de nivel (organización escolar), cambio en las expectativas familiares, cambio de materias y grado de dificultad de éstas y, finalmente, la consciencia, por parte de los padres, de lo que puede ser el destino de la propia vida de estudiante, ya que en Andorra no existe la posibilidad de cursar determinados estudios universitarios y hay que acudir a España o Francia. Ello conlleva determinadas decisiones a la hora de elegir los centros de estudio.

Las hipótesis que formulábamos ante los agentes implicados (AMPA, equipos directivos y psicopedagógicos) en torno al problema de las discontinuidades nos permitieron predecir las variaciones y actuaciones que se deben introducir en el contexto escolar, tratando de dar solución al problema de la discontinuidad escolar en Andorra. El desarrollo de la investigación aplicada de este proyecto nos permitió poner a prueba nuestras hipótesis y evaluar y observar las consecuencias de los cambios a introducir en los distintos grupos de la escuela. Sobre esta base empírica pudimos contrastar las hipótesis, implementar las propuestas de mejora y evaluar su solución.

Por su parte las investigaciones básicas mencionadas nos han permitido elaborar algunas monografías como «El niño de 0 a 6 años, pautas de educación» (1997), «Guía para la toma de decisiones responsables ante el consumo de alcohol para padres con hijos de 6 a 13 años» (2004) 0 el «Manual para periodistas sobre la protección de la infancia (2004)». 
Baumrind, D. (1996). Parenting. The discipline controversy revisited. Family Relations, 45, pp. 405-414.

Colom, A. (2005). Antropología y antropologías de la educación. En Antropología: Horizontes educativos. Granada: Ediciones Universidad de Granada.

Colom, A. y Janer, G. (1995). El modelo cultural en la construcción de la Antropología de la Educación. En Cuestiones de Antropología de la Educación. Barcelona: Ceac.

Colom, A. y Núñez, L. (2001). Teoría de la Educación. Madrid: Síntesis.

Colom, A. y Rincón Verdera, J. C. (2004). Epistemología neoidealista y fracaso fundacional del saber educativo. Teoría de la Educación. Revista Interuniversitária,16, pp. 19-48.

Fullat, 0. (1992). Filosofías de la Educación. Barcelona: CEAC.

Gervilla, E. (1990). La Escuela del Nacional-Catolicismo. Granada: Impredisur.

Gervilla, E. (1994). ¿Filosofía de la Educación hoy? ¿Para qué? Bordón, 3, pp. 307-314.

Gervilla, E. (2000a). Modelo axiológico de educación integral. Revista Española de Pedagogía, 215, pp. 39-58.

Gervilla, E. (2000b). Filosofía de la Educación Hoy. Necesidad de recuperar el sabor del saber y el sentido último de la Formación Humana. Revista de Ciencias de la Educación, 184, pp. 15-28 y Analecta Calasanctiana, 84, pp. 35-50.

Gervilla, E. (2004). Buscando valores. El análisis de contenido axiológico. Perfiles Educativos, 26 (103), pp. 95-111.

Geertz, C. (1990). Descripción densa: hacia una teoría interpretativa de la cultura. En G. Geertz. La interpretación de las culturas. Barcelona: Gedisa.

Goetz, J P. y Le Compte, M. D. (1988). Etnografía y diseño cualitativo en investigación educativa. Madrid: Morata.

Jacob, E. (1987). Qualitative research traditions: A review. Review of Educational Research 57, pp. $1-50$.

Maccoby, E. E., y Martín, J. A. (1983) Socialization in the context of the family: Parent-child interaction. En Müssen, P. H. y Hetherington, E. M. (eds.). Handbook of child psychology: Vol. 4. Socialization, personality, and social development (4th ed.). New York: Wiley, pp. 1-101.

Passmore,J. (1983). Filosofía de la enseñanza. México: FCE.

Peters, R. S. (1979). Filosofía de la educación. México: FCE.

Pérez Alonso-Geta, P. Ma. (1996). Valores y estilos de vida de la infancia en Andorra. Andorra: CONAPA.

Pérez Alonso-Geta, P. Ma (2001). Valores y pautas de crianza familiar en los Montes del Pas. Teoría de la Educación, 13,pp. 115-136. 
Pérez Alonso-Geta, P. Ma (2002). Valores y pautas de interacción familiar en la adolescencia 13-18 años. Madrid: S.M.

Pérez Alonso-Geta, P. Ma. (2003). El efecto socializador de la TV en niños y adolescentes de la Comunidad Valenciana. Valencia: Generalität Valenciana.

Pérez Alonso-Geta, R Ma y Cánovas Leonhardt, P. (1992). Valores y pautas de crianza familiar. Madrid: S. M.

Pérez Alonso-Geta, P. Ma; Marín, R. y Vázquez, G. (1992). Los valores de los niños españoles. Madrid: S. M.

Ritzer, G. (1993)- Teoría sociológica contemporánea. Madrid: Me Craw-Hill.

Rodríguez Neira, T. (1999a). La cultura contra la escuela. Barcelona: Ariel.

Rodríguez Neira, T. (1999b). Teorías y modelos de enseñanza. Posibilidades y límites. Lleida: Milenio.

Russell, B. (1972). Los problemas de la Filosofía. Barcelona: Labor.

Varios. (1989). Filosofía de la Educación Hoy: Conceptos, Autores, Temas. Madrid: Dykinson.

Varios. (1991). Filosofía de la Educación hoy. Autores: Selección de textos. Madrid: Dykinson.

Varios. (1997). Filosofia de la Educación hoy. Diccionario. Madrid: Dykinson.

Varios. (1998). Filosofía de la Educación hoy: Temas. Madrid: Dykinson.

Woods, R.G. y Barrow, R. ST. C. (1978). Introducción a la filosofía de la educación. Madrid: Anaya. 